\title{
Quantum duality and Bethe-Ansatz for the Hofstadter problem on hexagonal lattice
}

\author{
C.-A. Piguet, D.F. Wang and C. Gruber \\ Institut de Physique Théorique \\ Ecole Polytechnique Fédérale de Lausanne \\ PHB-Ecublens, CH-1015 Lausanne, Switzerland
}

\begin{abstract}
The Hofstadter problem is studied on the hexagonal lattice. We first establish a relation between the spectra for the hexagonal lattice and for its dual lattice, the triangular lattice. Following the idea of Faddeev and Kashaev, we then obtain the Bethe-Ansatz equations for this system.

PACS number: 71.30.+h, 05.30.-d, 74.65+n, 75.10.Jm
\end{abstract}

Typeset Using REVTEX 
Systems in external magnetic field have been of considerable interest in recent years. One of the most fascinating properties of these systems is the integer and fractional quantum Hall effect [1] 3]. The essential physics of the integer quantum Hall effect can be described by the simple Landau problem, in which a free electron moves in a two dimensional plane under constant magnetic field. For the fractional quantum Hall effect, it is well known that the electron-electron correlation gives rise to the energy gap of the system.

Besides the systems of electrons moving continuously on the two dimensional plane in an external magnetic field, the problem of free electrons hopping on a two dimensional lattice under external magnetic field, i.e. the Hofstadter problem, has attracted a lot of attention [11]. Recently, using reflection positivity, Lieb has provided a proof for the long standing conjecture that for the square lattice, the magnetic flux which minimises the energy of the system at half-filling is exactly $\pi$ per plaquette [10]. On the other hand, in their recent work, Wiegmann and Zabrodin have shown that the magnetic translations can be constructed with the generators of the quantum group $U_{q}(s l(2))$ for the Hofstadter problem [8]. With this representation, they obtain the Bethe-Ansatz equations for the eigenvalue problem of the system on a two dimensional square lattice. Later, Faddeev and Kashaev were able to provide a generalized approach, both for the square and triangular lattices [9].

In two dimensions, there are three lattices of special interest: the square, the triangular and the hexagonal lattices. In particular, the triangular lattice and the hexagonal lattice are dual of each other. In this paper, we study the Hofstadter problem on the hexagonal lattice. Following the idea of Wiegmann-Zabrodin, and Faddeev-Kashaev, we shall find the Bethe-Ansatz type solutions for this system. Furthermore, we establish a quantum duality relation between the energy spectra on the triangular and on hexagonal lattices.

The general Hamiltonian for non interacting electrons moving on a lattice in a magnetic field is:

$$
H=\sum_{i, j} t_{i j} e^{i \theta_{i j}} c_{i}^{+} c_{j}
$$

where $c_{i}^{+}, c_{i}$ are the creation and annihilation operators for an electron ai site $i, t_{i j}$ is the 
real hopping matrix, and $\theta_{i j}$ corresponds to $\int_{i}^{j} \vec{A}(\vec{x}) \cdot d \vec{x}$, with $\vec{A}$ the vector potential and the integral is performed on a straight line between $i$ and $j$. It thus satisfies $\theta_{i j}=-\theta_{j i}$. In the following, we consider the special case where the electrons hop between nearest neighbours only. We further assume that the system is invariant under translation, but not necessarily invariant under rotation.

The Hofstadter problem is a very interesting problem of theoretical physics, in particular due to its distinction between rational and irrational numbers. In the following, we only consider the rational case when the flux per elementary cell of the lattice is given by $\phi=$ $2 \pi \frac{M}{N}$, where $M$ and $N$ are mutually prime integers.

For the hexagonal lattice, we can define two triangular sublattices $A$ and $B$. Let $\vec{s}_{1}, \vec{s}_{2}$ and $\vec{s}_{3}$ denote the three vectors that connect a site of the sublattice $A$ with its three nearest neighbours of the sublattice $B$, chosen in such a way that $\vec{s}_{1} \wedge \vec{s}_{2}, \vec{s}_{2} \wedge \vec{s}_{3}$ and $\vec{s}_{3} \wedge \vec{s}_{1}$ are in the opposite direction of the magnetic field. We have three hopping amplitudes $t_{1}^{h}, t_{2}^{h}$ and $t_{3}^{h}$ corresponding to the three directions defined by $\vec{s}_{1}, \vec{s}_{2}$ and $\vec{s}_{3}$. The index $h$ recalls that we have a hexagonal lattice.

Let us consider a Bloch wavefunction with vector $\vec{k}\left(0 \leq k_{x}, k_{y} \leq \frac{2 \pi}{N}\right)$ :

$$
\left|\Psi>=\sum_{\vec{n}} e^{i \vec{k} \cdot \vec{n}} u_{\vec{n}} c_{\vec{n}}^{+}\right| 0>
$$

where the summation $\vec{n}$ is over the magnetic unit cell. We only need to consider the above region of the momentum, as in the rest of Brillouin zone, the situation can be mapped to this case.

With the Hamiltonian (1), the Schrödinger's equation yields the following equation for the coefficients $u_{\vec{n}}$ corresponding to the energy $E^{h}$ :

$$
\begin{aligned}
& E^{h} u_{\vec{n}}=\sum_{i=1}^{3} t_{i}^{h} \alpha_{i}^{h} e^{i \theta_{\vec{n}, \vec{n}+\vec{s}_{i}}} u_{\vec{n}+\vec{s}_{i}} \text { if } \vec{n} \in A \\
& E^{h} u_{\vec{n}}=\sum_{i=1}^{3} t_{i}^{h}\left(\alpha_{i}^{h}\right)^{-1} e^{i \theta_{\vec{n}, \vec{n}-\vec{s}_{i}}} u_{\vec{n}-\vec{s}_{i}} \text { if } \vec{n} \in B
\end{aligned}
$$

where $\alpha_{i}^{h}=e^{i \vec{k} \cdot \vec{s}_{i}}$. Combining (4) and (3), we obtain the eigenvalue equation for the coefficients $u_{\vec{n}}$ of the sublattice $A$ : 


$$
\begin{aligned}
& \left\{\left(E^{h}\right)^{2}-\left(\left(t_{1}^{h}\right)^{2}+\left(t_{2}^{h}\right)^{2}+\left(t_{3}^{h}\right)^{2}\right)\right\} u_{\vec{n}}= \\
& \sum_{i \neq j=1}^{3} t_{i}^{h} t_{j}^{h} \alpha_{i}^{h}\left(\alpha_{j}^{h}\right)^{-1} e^{i \theta_{\vec{n}, \vec{n}+\vec{s}_{i}}+i \theta_{\vec{n}+\vec{s}_{i}, \vec{n}+\vec{s}_{i}-\vec{s}_{j}}} u_{\vec{n}+\vec{s}_{i}-\vec{s}_{j}} .
\end{aligned}
$$

At this point, we define the three vectors $\vec{S}_{1}=\vec{s}_{2}-\vec{s}_{3}, \vec{S}_{2}=\vec{s}_{3}-\vec{s}_{1}$ and $\vec{S}_{3}=\vec{s}_{1}-\vec{s}_{2}$ which connect nearest neighbours of the sublattice $A$. Using the fact that:

$$
\theta_{\vec{n}, \vec{n}+\vec{s}_{i}}+\theta_{\vec{n}+\vec{s}_{i}, \vec{n}+\vec{s}_{i}-\vec{s}_{j}}=\epsilon_{i j k} \frac{\phi}{6}+\theta_{\vec{n}, \vec{n}+\epsilon_{i j k} \vec{S}_{k}}
$$

with $\epsilon_{i j k}$ the Levi-Civita symbol and $\phi$ the flux through elementary hexagons, we have:

$$
\begin{aligned}
& \left\{\left(E^{h}\right)^{2}-\left(\left(t_{1}^{h}\right)^{2}+\left(t_{2}^{h}\right)^{2}+\left(t_{3}^{h}\right)^{2}\right)\right\} u_{\vec{n}}= \\
& \sum_{i \neq j \neq k=1}^{3} t_{i}^{h} t_{j}^{h} \alpha_{i}^{h}\left(\alpha_{j}^{h}\right)^{-1} \omega^{\epsilon_{i j k} \frac{1}{6}} e^{i \theta_{\vec{n}, \vec{n}+\epsilon_{i j k} \vec{S}_{k}}} u_{\vec{n}+\epsilon_{i j k} \vec{S}_{k}},
\end{aligned}
$$

where $\omega=e^{i \phi}$.

Let us then consider the Hofstadter problem on the triangular lattice. With $t_{1}^{t}, t_{2}^{t}, t_{3}^{t}$ the three hopping amplitudes the Schrödinger's equation gives the following equation for the coefficients $u_{\vec{n}}$ corresponding to the energy $E^{t}$ :

$$
E^{t} u_{\vec{n}}=\sum_{i=1}^{3}\left(t_{i}^{t} \alpha_{i}^{t} e^{i \theta_{\vec{n}, \vec{n}+\vec{S}_{i}}} u_{\vec{n}+\vec{S}_{i}}+t_{i}^{t}\left(\alpha_{i}^{t}\right)^{-1} e^{i \theta_{\vec{n}, \vec{n}-\vec{S}_{i}}} u_{\vec{n}-\vec{S}_{i}}\right)
$$

where $\alpha_{i}^{t}=e^{i \vec{k} \cdot \vec{S}_{i}}$.

With the above relations, one can now easily relate the energies of the hexagonal lattice to the ones of the triangular lattice:

$$
\left(E^{h}\right)^{2}-\left(\left(t_{1}^{h}\right)^{2}+\left(t_{2}^{h}\right)^{2}+\left(t_{3}^{h}\right)^{2}\right)=E^{t}
$$

where we have the following correspondances of the hopping parameters and of the momenta:

$$
\begin{aligned}
t_{1}^{t} & =t_{2}^{h} t_{3}^{h} \\
t_{2}^{t} & =t_{3}^{h} t_{1}^{h} \\
t_{3}^{t} & =t_{1}^{h} t_{2}^{h} \\
\alpha_{1}^{t} & =\alpha_{2}^{h}\left(\alpha_{3}^{h}\right)^{-1} \omega^{\frac{1}{6}}
\end{aligned}
$$




$$
\begin{aligned}
& \alpha_{2}^{t}=\alpha_{3}^{h}\left(\alpha_{1}^{h}\right)^{-1} \omega^{\frac{1}{6}} \\
& \alpha_{3}^{t}=\alpha_{1}^{h}\left(\alpha_{2}^{h}\right)^{-1} \omega^{\frac{1}{6}} .
\end{aligned}
$$

It is well known that the spectrum of any bipartite lattice is symmetric around zero. This can be understood from the fact that the Hamiltonian $H$ can be transformed in $-H$ through the following unitary transformation:

$$
c_{x} \longrightarrow\left\{\begin{aligned}
c_{x} & \text { if } x \in A \\
-c_{x} & \text { if } x \in B
\end{aligned}\right.
$$

The hexagonal lattice is bipartite, while this is not the case for the triangular lattice. Therefore the spectrum on the hexagonal lattice is symmetric around zero and this property does not hold for the triangular lattice. This result is clearly seen with Eq.(9).

The duality relation expressed by Eq.(9) and Eq.(10) allow us to use directly the results of Faddeev and Kashaev [9] to find the Bethe-Ansatz equations for the hexagonal system. Let us recall briefly the results for the triangular lattice. Using a $N^{3}$ reducible representation, the Hilbert space is a tensor product of three subspaces, each of which has dimension $N$. A wavefunction can thus be written as

$$
|\phi>=| \phi>_{0} \otimes\left|\phi>_{1} \otimes\right| \phi>_{2} .
$$

Then, introduce the following notation:

$$
\begin{aligned}
& \alpha_{1}^{t}=e_{2}^{1 / 2}\left(f_{2} C\right)^{-1 / 2}, \alpha_{2}^{t}=e_{1}^{1 / 2}\left(f_{1} C\right)^{-1 / 2}, \alpha_{3}^{t}=e_{0}^{1 / 2}\left(f_{0} C\right)^{-1 / 2} \\
& t_{1}^{t}=\left(e_{2} f_{2} C\right)^{1 / 2}, t_{2}^{t}=\left(e_{1} f_{1} C\right)^{1 / 2}, t_{3}^{t}=\left(e_{0} f_{0} C\right)^{1 / 2} .
\end{aligned}
$$

One also has

$$
e_{i}=b_{i-1} d_{i} c_{i+1}, f_{i}=c_{i-1} a_{i} b_{i+1},
$$

with $i \in Z_{3}$. Here $C$ is a constant complex number, $C^{N}=(-1)^{(N-1)}$. 
The eigenvalue equation for the projection $Q(p)$ of an eigenvector $\mid \phi>$ on the Baxter's vector $\mid p>$, which corresponds to the point $p=\left(x, \xi_{0}, \xi_{1}, \xi_{2}\right)$ on a curve in a four dimensional space, reads

$$
\Lambda(x) Q(p)=Q\left(\tau_{-} p\right) \Delta_{-}(p)+Q\left(\tau_{+} p\right) \Delta_{+}(p), Q(p)=<\phi \mid p>
$$

where $\Lambda(x)$ is related to the eigenvalue $E^{t}$ by

$$
\Lambda(x)=\omega a_{0} a_{1} a_{2} C+d_{0} d_{1} d_{2}+x^{2} E^{t}
$$

$\tau_{+}$and $\tau_{-}$are defined on the coordinates $x, \xi_{0}, \xi_{1}, \xi_{2}$ by

$$
\tau_{ \pm} x=\omega^{ \pm 1 / 2} x, \tau_{ \pm} \xi_{i}=\omega^{-1 / 2} \xi_{i}, i=0,1,2
$$

and

$$
\begin{aligned}
& \Delta_{-}(p)=\prod_{i \in Z_{3}}\left(d_{i}-x \xi_{i+1} c_{i}\right) \\
& \Delta_{+}(p)=\prod_{i \in Z_{3}} \xi_{i}\left(a_{i} d_{i}-x^{2} b_{i} c_{i}\right) /\left(\xi_{i+1} a_{i}-x b_{i}\right) .
\end{aligned}
$$

The zeros $p_{k}$ of $Q(p)$ are given by the following Behte-Ansatz equation

$$
\frac{Q\left(\tau_{-} p_{k}\right)}{Q\left(\tau_{+} p_{k}\right)}=-\frac{\Delta_{+}\left(p_{k}\right)}{\Delta_{-}\left(p_{k}\right)} .
$$

The equations for the hexagonal lattice take the same form except that one has to introduce (10) into the definitions (13).

In the special case where the genus of the curve vanishes and $N$ is odd $(N=2 P+1)$, one may simplify the above equations, so that they can be written out explicitly. This special case corresponds to the values of momenta: $\alpha_{1}^{t}=\alpha_{2}^{t}=\alpha_{3}^{t}=q^{1 / 2}$, with $q=\omega^{1 / 2}$. The energy for the hexagonal lattice reads:

$$
\begin{aligned}
\left(E^{h}\right)^{2}= & \left(t_{1}^{h}\right)^{2}+\left(t_{2}^{h}\right)^{2}+\left(t_{3}^{h}\right)^{2}+\left(q^{1 / 2}+q^{-1 / 2}\right)\left(t_{2}^{h} t_{3}^{h}+t_{3}^{h} t_{1}^{h}+t_{1}^{h} t_{2}^{h}\right) \\
& -\left(q-q^{-1}\right)\left(\left(t_{1}^{h}\right)^{2} t_{2}^{h} t_{3}^{h}+t_{1}^{h}\left(t_{2}^{h}\right)^{2} t_{3}^{h}+t_{1}^{h} t_{2}^{h}\left(t_{3}^{h}\right)^{2}\right) \sum_{m=1}^{2 P} z_{m} \\
& +\left(t_{1}^{h} t_{2}^{h} t_{3}^{h}\right)^{2}\left(q-q^{-1}\right)\left(q^{1 / 2}-q^{-1 / 2}\right) \sum_{1 \leq m<n \leq 2 P} z_{m} z_{n}
\end{aligned}
$$


where the $z_{l}(l=1, \ldots, 2 P)$ are given by the Bethe-Ansatz equations:

$$
\prod_{m=1, m \neq l}^{2 P} \frac{q z_{l}-z_{m}}{z_{l}-q z_{m}}=q^{-1 / 2} \frac{\left(t_{2}^{h} t_{3}^{h} z_{l}+q^{1 / 2}\right)\left(t_{3}^{h} t_{1}^{h} z_{l}+q^{1 / 2}\right)\left(t_{1}^{h} t_{2}^{h} z_{l}+q^{1 / 2}\right)}{\left(q^{1 / 2} t_{2}^{h} t_{3}^{h} z_{l}-1\right)\left(q^{1 / 2} t_{3}^{h} t_{1}^{h} z_{l}-1\right)\left(q^{1 / 2} t_{1}^{h} t_{2}^{h} z_{l}-1\right)} .
$$

In summary, we have developed the Bethe-Ansatz for the Hofstadter problem on the hexagonal lattice in this paper. An interesting duality is discovered between the hexagonal lattice and its dual partner (triangular lattice). Using this duality relation, we have written the Behte-Ansatz equations for the point of the magnetic Brillouin zone where the equations of Faddeev and Kashaev take an explicit form. Further work is necessary to obtain more information from these equations.

We wish to thank Prof. H. Kunz, Prof. F. Reuse, Prof. S. Maumary, Prof. Y. S. Wu, Prof. Mo-lin GE and Prof. E. H. Lieb for conversations. This work was supported in part by the Swiss National Science Foundation. 


\section{REFERENCES}

[1] V. Kalmeyer, R. B. Laughlin, Phys. Rev. Lett. 59, 2095 (1987).

[2] The Quantum Hall Effect, edited by R. Prange and S. Girvin (Springer-Verlag, 1990) ( general references therein ).

[3] Z. Zou, B. Doucot and B. S. Shastry, Phys. Rev B 39, 11424 (1989).

[4] D. J. Thouless, Phys. Rev. B 28, 4272 (1983).

[5] P. G. Harper, Proc. Phys. Soc. London, Sect. A 68, 872 (1955).

[6] J. B. Sokoloff, Phys. Rev. B 23, 2039 (1981).

[7] D. R. Hofstadter, Phys. Rev. B 14, 2239 (1976).

[8] P. B. Wiegmann, A. V. Zabrodin, Phys. Rev. Lett. 72, 1890 (1994).

[9] L. D. Fadeev, R. M. Kashaev, Commun. Math. Phys. 169, 181 (1995).

[10] E. H. Lieb, Phys. Rev. Lett. 73, 2158 (1994), Helv. Phys. Acta 65, 247 (1992).

[11] J. Zak Phys. Rev. 134, 1602 (1964). 